OPEN ACCESS

Edited by:

Max Maurin,

Université Grenoble Alpes,

France

Reviewed by:

Heer Mehta,

Rice University, United States

Vincent Cattoir,

University of Rennes 1, France

*Correspondence:

Floriana Campanile

f.campanile@unict.it

${ }^{+}$These authors have contributed equally to this work and share first authorship

Specialty section:

This article was submitted to Clinical Microbiology,

a section of the journal

Frontiers in Cellular and

Infection Microbiology

Received: 17 November 2021 Accepted: 23 December 2021

Published: 20 January 2022

Citation:

Lazzaro LM, Cassisi M, Stefani S and Campanile $F$ (2022) Impact of PBP4 Alterations on $\beta$-Lactam Resistance and Ceftobiprole NonSusceptibility Among Enterococcus faecalis Clinical Isolates. Front. Cell. Infect. Microbiol. 11:816657. doi: 10.3389/fcimb.2021.816657

\section{Impact of PBP4 Alterations on $\beta$-Lactam Resistance and Ceftobiprole Non-Susceptibility Among Enterococcus faecalis Clinical Isolates}

\author{
Lorenzo M. Lazzaro ${ }^{\dagger}$, Marta Cassisi ${ }^{\dagger}$, Stefania Stefani and Floriana Campanile * \\ Section of Microbiology, Department of Biomedical and Biotechnological Sciences (BIOMETEC), Microbiologia Medica \\ Molecolare e Antibiotico Resistenza (MMARLab), University of Catania, Catania, Italy
}

Penicillin-resistance among Enterococcus faecalis clinical isolates has been recently associated with overexpression or aminoacidic substitutions in low-affinity PBP4. Ceftobiprole (BPR), a new-generation cephalosporin, is a therapeutic option against $E$. faecalis. Here, we present evidence that pbp4 gene sequence alterations may influence the expression level of the gene and ceftobiprole binding to PBP4 in E. faecalis clinical isolates showing remarkable MDR-phenotypes, and how this could interfere with BPR in vitro antibacterial and bactericidal activity. Seven E. faecalis strains from bloodstream infections were analyzed for their antibiotic and $\beta$-lactam resistance. BPR bactericidal activity was assessed by time-kill analysis; $p b p 4$ genes were sequenced and pbp4 relative expression levels of transcription were performed by RT-qPCR. Five penicillin-resistant ampicillin-susceptible (PRAS) isolates were detected, 4 of which were also BPR nonsusceptible (BPR-NS). In the time-kill experiments, BPR exposure resulted in a potent bactericidal activity (3-5 $\log _{10}$ reduction) at the different concentrations tested. pbp4 gene sequence analysis revealed some mutations that may account for the changes in PBP4 affinity and MIC increase in the 4 BPR-NS strains (MICs 4-16 mg/L): the deletion of an adenine (de/A) in the promoter region in all PRAS/BPR-NS strains; 12 different amino acid substitutions, 7 of which were next to the PBP catalytic-sites. The most significant were: T418A, located 6 amino acids (aa) upstream of the catalytic-serine included in the ${ }^{424}$ STFK $^{427}$ motif I; L475Q, 7 aa upstream of the ${ }^{482} \mathrm{SDN}^{484}$ motif II; V606A and the novel $\mathrm{Y} 605 \mathrm{H}, 13 / 14$ aa upstream of the ${ }^{619} \mathrm{KTGT}^{622}$ motif III. Taken together, our data showed that elevated BPR MICs were attributable to increased transcription of pbp4 associated with a single upstream adenine deletion and PBP4 alterations in the catalyticsite motifs - which might interfere with the formation of the BPR/PBP4 complex. pbp4 molecular alterations may account for the changes in PBP4 affinity and MIC increase, 
without affecting BPR cidal activity. Indeed, our in vitro dynamic analysis by time-kill assays showed that BPR exerted a bactericidal activity against $E$. faecalis clinical isolates, despite their MDR phenotypes.

Keywords: Enterococcus faecalis, time-kill curve assays, PBP4, ceftobiprole, pbp4 gene expression

\section{INTRODUCTION}

Enterococci are the third most commonly isolated nosocomial pathogens, accounting for $12 \%$ of all hospital infections (Hollenbeck and Rice, 2012). The clinical importance of the genus Enterococcus is closely related to antibiotic resistance, which contributes to the risk of infection.

Enterococci have high-level resistance to most cephalosporins and all semi-synthetic penicillins.

Among the species of greatest clinical interest, E. faecalis is intrinsically resistant to most $\beta$-lactams and only susceptible to a limited group of penicillins, such as ampicillin, penicillin and piperacillin (Arias and Murray, 2012; Kristich et al., 2014a).

Ampicillin resistance has been rarely reported in E. faecalis, as this did not represent a clinical and therapeutic challenge. Until recently, it was assumed that ampicillin- susceptible $E$. faecalis was also susceptible to penicillin, but $E$. faecalis clinical isolates have been exhibiting increasing levels of resistance to penicillin, due to the emergence of Penicillin-Resistant Ampicillin-Susceptible (PRAS) isolates, eliminating $\beta$-lactams as a treatment option. This uncommon phenotype has been reported in various hospitals worldwide but its real epidemiological impact is still unknown (Metzidie et al., 2006; Guardabassi et al., 2010; Tan et al., 2014; Cabrera et al., 2020; Conceição et al., 2020; Gawryszewska et al., 2021).

Reduced susceptibility to $\beta$-lactams in E. faecalis is attributable to two main mechanisms: the first is the production of $\beta$-lactamases, rarely described among E. faecalis strains (Rice and Murray, 1995; Sarti et al., 2012; Schell et al., 2020), while the second is the overproduction of a single low-affinity class $B$ penicillin-binding protein (PBP), named PBP4. The PBP4 active site in the Trans-Peptidase (TPase) domain encompasses three conserved motifs: the ${ }^{424} \mathrm{STFK}^{427}$ motif I, containing the catalytic serine; the ${ }^{482} \mathrm{SDN}^{484}$ motif II, involved in the protonation of the $\beta$-lactam leaving group; and the ${ }^{619} \mathrm{KTGT}^{622}$ motif III, which facilitates substrate binding and defines the oxyanion hole (Ghuysen, 1991; Djoric et al., 2020). Accumulation of point mutations in the penicillin-binding module of PBP4 has been associated with a decreased affinity for $\beta$-lactams (Ono et al., 2005; Zapun et al., 2008; Infante et al., 2016; Moon et al., 2018; Rice et al., 2018; Gawryszewska et al., 2021).

The pandemic led to an alarming increase of E. faecalis isolated from patients with COVID-19 under mechanical ventilation and ICU-acquired enterococcals BSI (Giacobbe et al., 2021; Posteraro et al., 2021), also worsened by their increasing multi-resistance to all therapeutic options. E. faecalis pathogens play a crucial role in determining the severity of the clinical conditions, critically influencing the patients' outcome, and represent a serious threat in infection therapy (Kim et al., 2019).

Among $5^{\text {th }}$ generation cephalosporins, ceftobiprole exerts superior in vitro antibacterial and bactericidal activity also against vancomycin-resistant and $\beta$-lactamase producing strains, due to its high affinity for PBPs (Mendes et al., 2016; Hamilton et al., 2017; Campanile et al., 2019).

The aims of this study were: 1) to investigate the in vitro antibacterial and bactericidal activity of BPR alone against E. faecalis clinical isolates belonging to selected antibiotic-resistance classes; 2) to analyze the occurrence of $p b p 4$ mutations and verify their role in influencing the activity of $\beta$-lactams and, specifically, of BPR; 3 ) to compare the $p b p 4$ expression levels in all E. faecalis clinical isolates with reduced susceptibility to beta-lactams and PBP4 alterations, with the aim of evaluating which of these alterations may be involved in non-susceptibility and BPR cidal activity, and how.

\section{MATERIALS AND METHODS}

\section{Strains}

Seven E. faecalis clinical strains, isolated from bloodstream infections (BSI) in Italian hospitals, were selected for their antibiotic-resistance behaviors from a larger collection of twentytwo isolates already characterized (Campanile, et al. 31st ECCMID 2021, P 2004). They belonged to the major MDR phenotypes (PRAS, BPR-NS, VRE, HLAR); two beta-lactam-susceptible E. faecalis isolates were also selected for comparison.

E. faecalis OG1RF, deposited in the American Type Culture Collection (ATCC) under ATCC 47077, deriving from E. faecalis OG1 by selection for resistance to rifampin and fusidic acid (Bourgogne et al., 2008), was used as control in molecular studies. E. faecalis ATCC 29212 was used as control for antibioticsusceptibility tests (EUCAST, 2021).

\section{Antimicrobial Susceptibility Testing}

Ceftobiprole was provided by Basilea Pharmaceutica International Ltd. (Basel, Switzerland); ceftaroline, linezolid and tigecycline by Pfizer Inc. (New York, NY, USA); daptomycin by Novartis (Basel, Switzerland). Penicillin, ampicillin, amoxicillin, imipenem, vancomycin, teicoplanin, gentamicin and streptomycin were purchased commercially (Sigma Chemical Co., ST. Louis, MO, USA). MICs were determined by broth microdilution and interpreted according to the European Committee on Antimicrobial Susceptibility Testing (EUCAST) clinical breakpoints (http://www.eucast.org/clinical_breakpoints/) (EUCAST, 2021). In the absence of EUCAST clinical breakpoints, those of the Clinical and Laboratory Standards Institute were applied (Clinical and Laboratory Standards Institute, 2021).

\section{Bactericidal Assays}

In vitro time-kill experiments were performed in duplicate in $20 \mathrm{~mL}$ tubes containing Cation Adjusted Mueller-Hinton broth (CA-MHB) 
(Difco, Detroit, MI) using a starting inoculum of $10^{5}-10^{6} \mathrm{CFU} / \mathrm{mL}$ with ceftobiprole (1X, 2X and $4 \mathrm{X}$ MIC). Bactericidal activity was defined as a $\geq 3 \log _{10}$ decrease in bacterial count at $24 \mathrm{~h}$ (White et al., 1996). Statistical analysis was performed using GraphPad Prism (Version 8.4.0). All experiments were performed in triplicate. Data were represented as mean $\pm \mathrm{SD}$ of triplicate experiments.

\section{Gene Amplification and Sequence Analysis} All isolates were molecularly characterized for the $p b p 4$ gene sequence in order to analyze possible mutations and verify their role in influencing BPR activity. pbp 4 was amplified by PCR and the entire gene was double-strand sequenced using oligonucleotides specifically designed for this study (Supplemental Table 1). Sequencing was performed using the Dye Terminator DNA sequencing kit V1.1 (Applied Biosystems TM), followed by purification using the DyeEx 2.0 Spin Kit (Quiagen, Hilden, Germany). The sequences obtained were corrected and analyzed using the Chromas Lite 2.1 program and then exported in FASTA format. Sequence alignment and gene and translated protein analysis were performed by using BLAST tool (Basic Local Alignment Search Tool) (https://blast.ncbi.nlm.nih.gov/Blast.cgi), CLC Sequence Viewer 8.0 and UniProt (www.uniprot.org). E. faecalis ATCC 47077, whose complete genome sequence is deposited at NCBI under the accession number CP025020.1., was used as reference.

\section{Real-Time Quantitative PCR}

For real-time quantitative PCR (RT-qPCR) studies, $7 \mathrm{~mL}$ of bacterial suspensions $\left(10^{5} \mathrm{CFU} / \mathrm{mL}\right)$ were incubated at $37^{\circ} \mathrm{C}$ until late-logphase $\left(0.1 \mathrm{OD}_{600} \cong 1 \times 10^{8} \mathrm{CFU} / \mathrm{mL}\right)$; total RNA was extracted using the RNeasy ${ }^{\circledR}$ Mini kit (Qiagen, Hilden, Germany), purified from contaminating DNA genomics and retro-transcripted in cDNA using the QuantiNova ${ }^{\mathrm{TM}}$ Reverse Transcription Kit (Qiagen, Hilden, Germany), according to the manufacturer's instructions. cDNA was quantified using the Qubit ${ }^{\mathrm{TM}} 4$ fluorometer.

RT-qPCR was performed in a Rotor-Gene Q (Qiagen, Hilden, Germany) instrument, using the QuantiNova ${ }^{\mathrm{TM}} \mathrm{SYBR}^{\circledR}$ Green PCR kit (Qiagen, Hilden, Germany), according to the manufacturer's instructions. pbp4 (5RT) and 16S rRNA qPCR oligonucleotides were specifically designed for this study (Supplemental Table 1). For each sample, three biological replicates were prepared. Relative gene expression levels of transcription were calculated by the quantification cycle (Cq) method and normalized to the expression of $16 \mathrm{~S}$ rRNA. Relative expression was calculated using the $2^{-\Delta \Delta C t}$ method (Livak and Schmittgen, 2001). The data obtained were expressed as the fold-change in expression compared to that of the ATCC 47077 reference. Comparison of the expression levels of transcription of all strains and statistical analysis were conducted using the Relative Expression Software Tool "REST 2009" (Qiagen, Hilden, Germany) and GraphPad Prism (Version 8.4.0).

\section{RESULTS}

\section{In Vitro Antibacterial Activity}

Table 1 shows the susceptibility values of the 7 E. faecalis strains in study to $\beta$-lactams and comparator drugs. Five strains were found to be penicillin-resistant ampicillin-susceptible (PRAS), besides showing reduced susceptibility to ceftaroline (MICs $\geq 4 \mathrm{mg} / \mathrm{L}$ ). Four out of 5 strains showed higher ceftobiprole MIC values $(\geq 4$ $\mathrm{mg} / \mathrm{L}$ ) and were reported as ceftobiprole non-susceptible (BPRNS). All isolates were also susceptible to the other $\beta$-lactams tested (amoxicillin and imipenem), and susceptible to daptomycin, linezolid and tigecycline. High-level resistance to gentamicin (HLGR) $(n=1)$, streptomycin (HLSR) $(n=1)$ and both aminoglycosides (HLAR) $(n=4)$ was detected. Vancomycin and teicoplanin resistance (VRE) was detected in 2 isolates and further found to be associated with the presence of the van $\mathrm{A}$ gene.

\section{Bactericidal Activity of Ceftobiprole}

In BPR-NS, BPR exposure resulted in a potent bactericidal activity ( 3 to $5 \log _{10}$ ) at 4 X MIC after $24 \mathrm{~h}$. BPR-S strains showed a greater log reduction ( 3 to $5 \log _{10}$ ) even at lower concentrations (i.e., $1 \mathrm{X}$ and/or 2X MIC). Enhanced killing activity was also observed at $8 \mathrm{~h}$ (Figure 1).

\section{pbp4 Sequence Analysis and Significant Protein Alterations}

PCR and sequence analysis of the $p b p 4$ gene revealed some mutations that may account for the changes in PBP4 affinity and MIC increase in the 4 BPR-NS strains (MICs $4-16 \mathrm{mg} / \mathrm{L}$ ). The adenine deletion ( $\operatorname{del} \mathrm{A})$ in the promoter region, $8 \mathrm{bp}$ upstream of the -35 consensus site, was the carried by all 4 strains showing non-susceptibility to BPR and high-level resistance to penicillin (Tables 1, 2). Sequence analysis of translated PBP4 identified 12 different missense mutations, 7 of which were next to the PBP catalytic sites (Table 2; Figure 2). Notably, the T418A mutation (Efs1) was located 6 amino acids (aa) upstream of the catalytic serine included in the ${ }^{424} \mathrm{STFK}^{427}$ motif I; L475Q (Efs8), 7 aa upstream of the ${ }^{482} \mathrm{SDN}^{484}$ motif II, involved in $\beta$-lactam leaving group protonation; the novel Y605H (Efs18) and V606A (Efs7), $13 / 14$ aa upstream of the ${ }^{619} \mathrm{KTGT}^{622}$ motif III, which facilitates substrate binding. Other mutations were found far from the PBP catalytic site: i) T50I, quite common in our sample (3/7), and I223V, only present in a fully susceptible strain (Efs20) in which it does not affect MICs to $\beta$-lactams, both located in the $\mathrm{N}$ terminal end; ii) L639F, T665I and T678A (all in Efs7), and D666P (Efs1), located in the C-terminal end.

Two further mutations in the region between the ${ }^{482} \mathrm{SDN}^{484}$ and ${ }^{619} \mathrm{KTGT}^{622}$ catalytic sites were detected (A536T; D573E) in Efs 1 and Efs11, respectively; in Efs11, the D573E substitution potentially affects the MIC values of penicillin and ceftaroline (16 and $32 \mathrm{mg} / \mathrm{L}$, respectively), but not those of ceftobiprole ( $2 \mathrm{mg} / \mathrm{L}$ ).

Nucleotide Sequence Accession Numbers. The complete sequences of the pbp4 gene variants have been deposited in GenBank under accession numbers from OM032878 to OM032884.

\section{Increase in the Level of pbp4 Expression}

The evaluation of $p b p 4$ gene expression relative to that of the ATCC47077 (OG1RF) reference strain showed varying levels of upregulation in all strains, linked to their $\beta$-lactam MIC values. The PRAS/BPR-S Efs2 and Efs20 strains exhibited lower expression levels $\left(\leq 10^{2}\right.$ fold-change increase). All PRAS-BPR-NS 
A
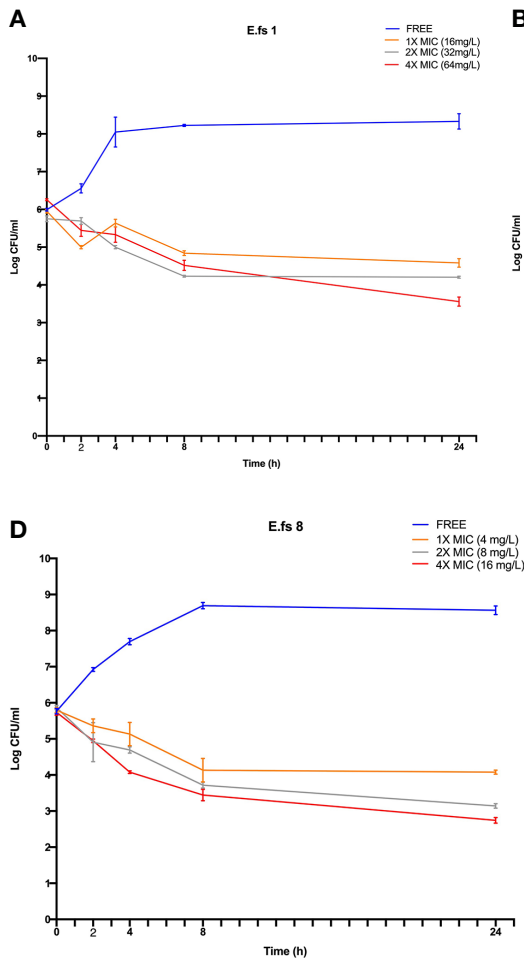

B

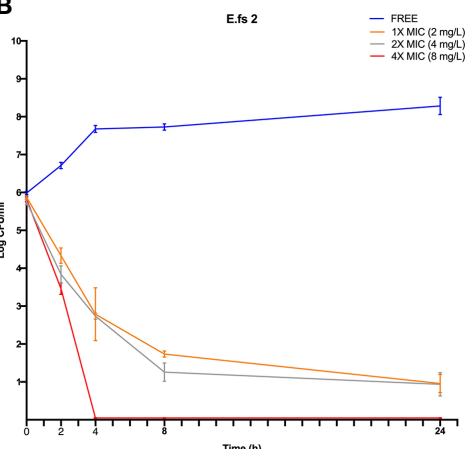

Time (n)
C
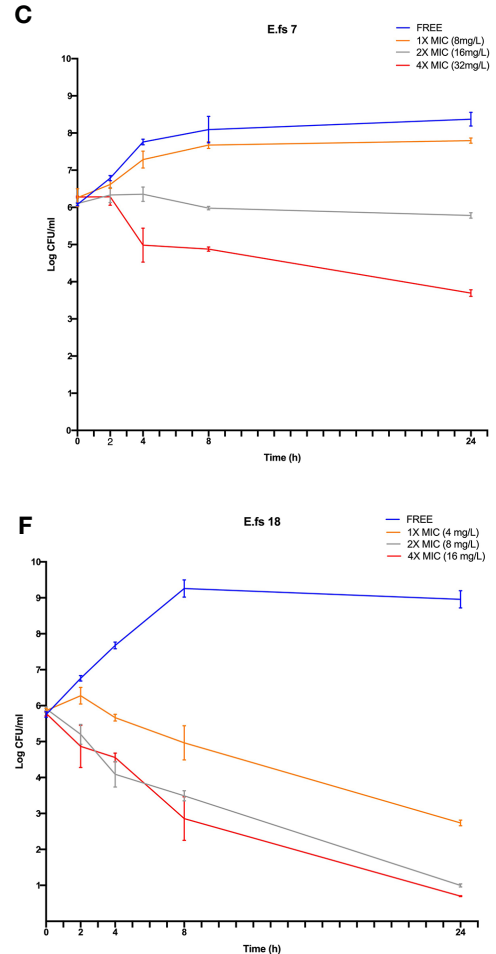

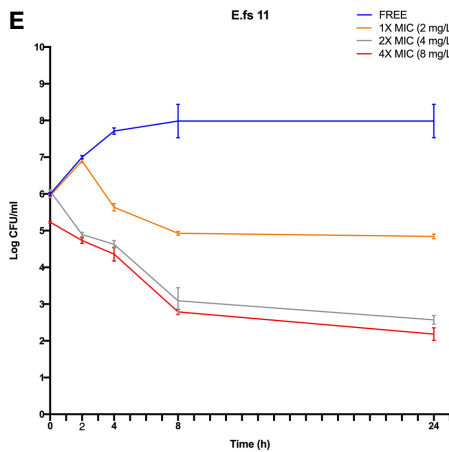

(1)

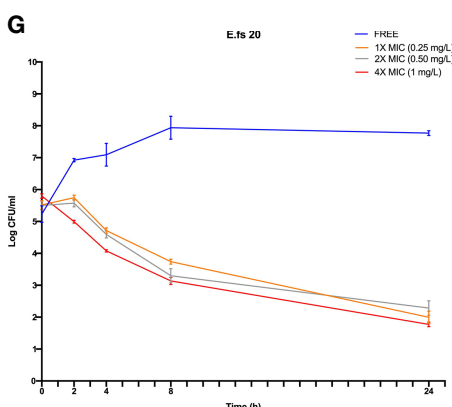

FIGURE 1 | Time-kill assays of ceftobiprole (BPR) against the seven E. faecalis clinical isolates in study. Cell count was reported as $\log _{10}$ (CFU/ml) at $0,2,4,8$ and 24h Time-points (T0, T2, T4, T24); Ceftobiprole (BPR) exposure was tested at 1X, 2X and 4X MICs. Error bars represent standard deviations ( \pm SD) of the mean of triplicate experiments (A-G)

TABLE 1 | Beta-lactams and comparator antimicrobial MIC values against E. faecalis clinical isolates.

\begin{tabular}{|c|c|c|c|c|c|c|c|c|c|c|c|c|c|}
\hline \multirow[t]{2}{*}{ Code } & \multicolumn{13}{|c|}{ MIC values (mg/L) } \\
\hline & $\mathbf{P}^{1}$ & AMP & AML & IMI & BPR $^{2}$ & $\mathrm{CPT}^{2}$ & VA & TEC & $\mathrm{CN}$ & $\mathbf{s}$ & LNZ & TGC & DAP \\
\hline Efs1 & 16 & 1 & 0.5 & 4 & 16 & $>256$ & 0.5 & 2 & $>1024$ & $>1024$ & 4 & 0.25 & 0.5 \\
\hline Efs7 & 64 & 4 & 4 & 4 & 8 & $>256$ & 1 & 2 & $>1024$ & $>1024$ & 4 & 0.06 & 0.5 \\
\hline Efs8 & 16 & 4 & 1 & 2 & 4 & 32 & $>256$ & $>256$ & $>1024$ & 128 & 4 & 0.125 & 0.5 \\
\hline Efs11 & 16 & 4 & 1 & 4 & 2 & 32 & $>256$ & 128 & $>1024$ & $>1024$ & 2 & 0.25 & 0.5 \\
\hline
\end{tabular}

${ }^{1}$ Penicillin and Daptomycin susceptibility values were established according to CLSI breakpoints (EUCAST breakpoints absent). ${ }^{2}$ Ceftobiprole and Ceftaroline: No EUCAST and CLSI official breakpoints; eCOFFs not determined.

P, Penicillin; AMP, Ampicillin; AML, Amoxicillin; IMI, Imipenem; BPR, Ceftobiprole; CPT, Ceftaroline; VA, Vancomycin; TEC, Teicoplanin; CN, Gentamicin; S, Streptomycin; LNZ, Linezolid; TGC, Tigecyclin; DAP, Daptomycin. 
TABLE 2 | Ceftobiprole MIC values (mg/L) and expression levels for E. faecalis clinical isolates, compared to sequence alterations.

\begin{tabular}{|c|c|c|c|c|c|c|c|c|c|c|c|c|c|c|c|c|}
\hline \multirow[t]{3}{*}{ Code } & \multirow{3}{*}{$\begin{array}{c}\text { Phenotype } \\
\text { characteristics }\end{array}$} & \multirow{3}{*}{$\begin{array}{l}\text { BPR MIC } \\
(\mathrm{mg} / \mathrm{L})^{a}\end{array}$} & \multirow{3}{*}{$\begin{array}{l}\text { §Fold-change } \\
\text { mean }\end{array}$} & \multirow{3}{*}{$\begin{array}{l}\text { Deletion in promoter } \\
\text { region }^{\mathbf{b}}\end{array}$} & \multicolumn{12}{|c|}{ Amino acid substitutions in PBP4 ${ }^{d}$} \\
\hline & & & & & \multicolumn{8}{|c|}{ PBP active-sites } & \multirow[b]{2}{*}{$639 \mathrm{~L}$} & \multirow[b]{2}{*}{$665 \mathrm{~T}$} & \multirow[b]{2}{*}{${ }_{666} \mathrm{D}$} & \multirow[b]{2}{*}{$678 \mathrm{~T}$} \\
\hline & & & & & ${ }_{50} \mathrm{~T}$ & $223 \mathrm{l}$ & $418 \mathrm{~T}$ & $475 \mathrm{~L}$ & ${ }_{536} \mathrm{~A}$ & ${ }_{573} \mathrm{D}$ & $605 Y$ & $606 V$ & & & & \\
\hline Efs20 & PSAS; BPR-S; HLSR & 0.25 & 88.80 & - & - & $\mathbf{v}$ & - & - & - & - & - & - & - & - & - & - \\
\hline Efs2 & $\begin{array}{l}\text { PSAS; BPR-S; fully } \\
\text { susceptible }\end{array}$ & 2 & 77.36 & - & - & - & - & - & - & - & - & - & - & - & - & - \\
\hline Efs11 & $\begin{array}{l}\text { PRAS; BPR-S; VRE/ } \\
\text { vanA; HLAR }\end{array}$ & 2 & 695.413 & - & - & - & - & - & - & $\mathbf{E}$ & - & - & - & - & - & - \\
\hline Efs8 & $\begin{array}{l}\text { PRAS; BPR-NS; VRE/ } \\
\text { vanA; HLGR }\end{array}$ & 4 & 4851.96 & $\begin{array}{c}2013028 \_2013029 \\
\text { delA }^{c}\end{array}$ & 1 & - & - & $\mathbf{Q}$ & - & - & - & - & - & - & - & - \\
\hline Efs18 & PRAS; BPR-NS; HLAR & 4 & 422.88 & $\begin{array}{c}2013028 \_2013029 \\
\text { delA }^{c}\end{array}$ & - & - & - & - & - & - & $\mathbf{H}$ & - & - & - & - & - \\
\hline Efs7 & PRAS; BPR-NS; HLAR & 8 & 571.068 & $\begin{array}{c}2013028 \_2013029 \\
\text { delA }^{c}\end{array}$ & 1 & - & - & - & - & - & - & A & $\mathbf{F}$ & I & - & A \\
\hline Efs1 & PRAS; BPR-NS; HLAR & 16 & 698.895 & $\begin{array}{c}2013028 \_2013029 \\
\text { delA }\end{array}$ & $\mathbf{I}$ & - & $\mathbf{A}$ & - & $\mathbf{T}$ & - & - & - & - & - & $\mathbf{P}$ & - \\
\hline
\end{tabular}

${ }^{a} B P R$, Ceftobiprole; ${ }^{b}$ a single base pair deletion 8 bases upstream of the putative -35 region; ${ }^{c}$ Accession number GenBank: CP025020.1 (ATCC47077); ${ }^{d}$ Protein ID GenBank:

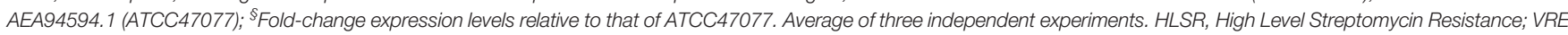
Vancomycin Resistant E. faecalis; HLAR, High Level Aminoglycosides Resistance; PRAS, Penicillin-Resistant Ampicillin-Susceptible; BPR-NS, Ceftobiprole Non-Susceptible; PSAS, Penicillin-Susceptible Ampicillin-Susceptible; BPR-S, Ceftobiprole Susceptible; HLGR, High Level Gentamicin Resistance. GenBank accession no. from OM032878 to OM032884.

A

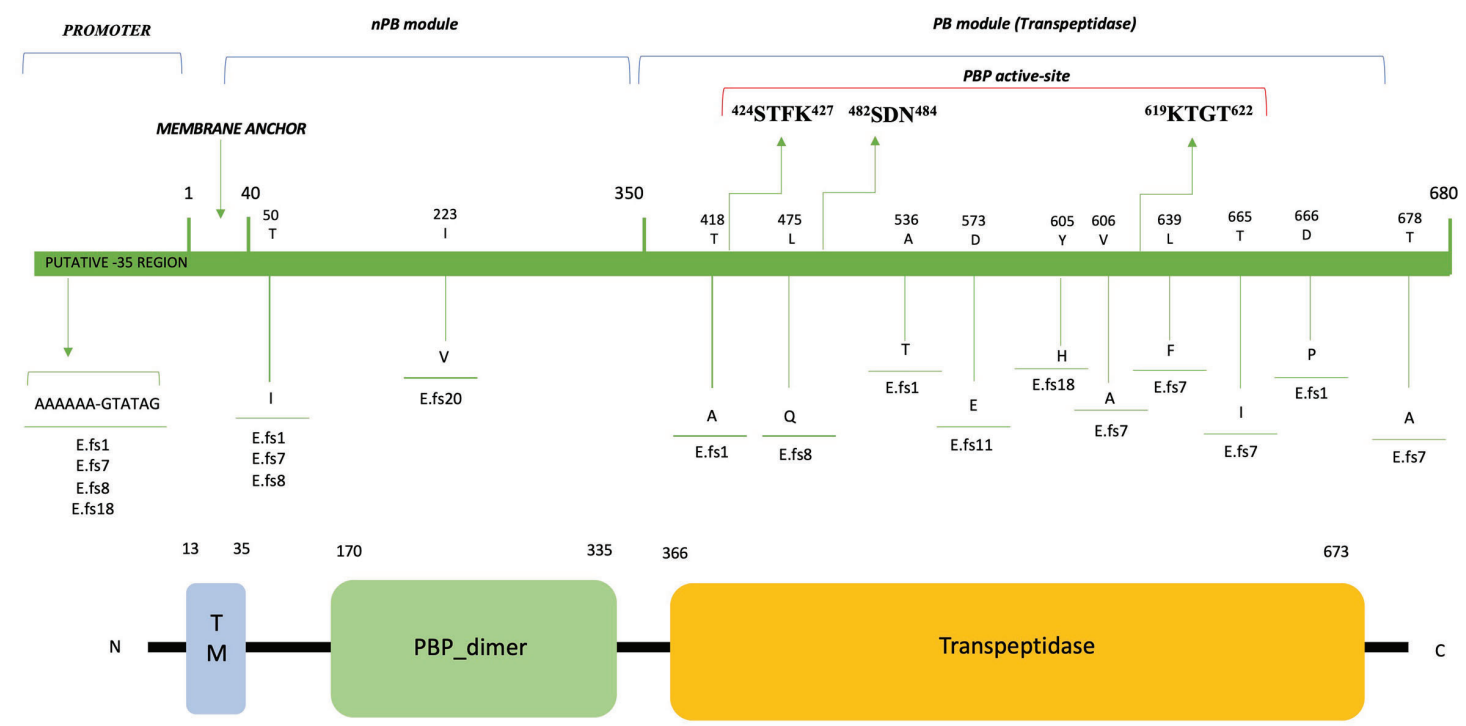

FIGURE 2 | Amino acid substitutions and domain architecture of PBP4 of E faecalis clinical isolates. GenBank accession no. from OM032878 to OM032884. The numbers above the diagrams indicate residue numbers of the domain boundaries in the proteins. (A) Non-Penicillin Binding module (nPB); Penicillin Binding module (PB); TM, transmembrane helix region. ${ }^{424} \mathrm{STFK}^{427},{ }^{482} \mathrm{SDN}^{484}$ and ${ }^{619} \mathrm{KTGT}^{622}$ : catalytic-site motifs (STFK 424 includes the catalytic serine $\mathrm{S}_{424}$ ). (B) The domain architecture of PBP4 of E faecalis ATCC 47077 (OG1RF) was analyzed using SMART (Simple Modular Architecture Research Tool; http://smart.embl-heidelberg.de/) and reported in a simplified version.

strains carrying the delA in the pbp4 promotor region and mutations in the PBP catalytic sites displayed $p b p 4$ overexpression with greater fold-change increases $\left(0.5-4 \times 10^{3}\right)$.

We observed that $p b p 4$ gene expression was more evident in the VRE/vanA strains (PRAS/BPR-NS Efs8 and PRSA/BPR-S Efs11), with or without delA in the promoter region (Figure 3, pattern-filled bars).

\section{Correlation Between Sequence Alterations and Expression Level Increase}

Missense mutations in the $p b p 4$ sequence were common in BPRNS and fully susceptible strains.

We observed a strong association between the increase in the level of $p b p 4$ expression and the adenine deletion in the promoter region, upstream of the coding sequence. delA leads to a $3 \log _{10}$ 


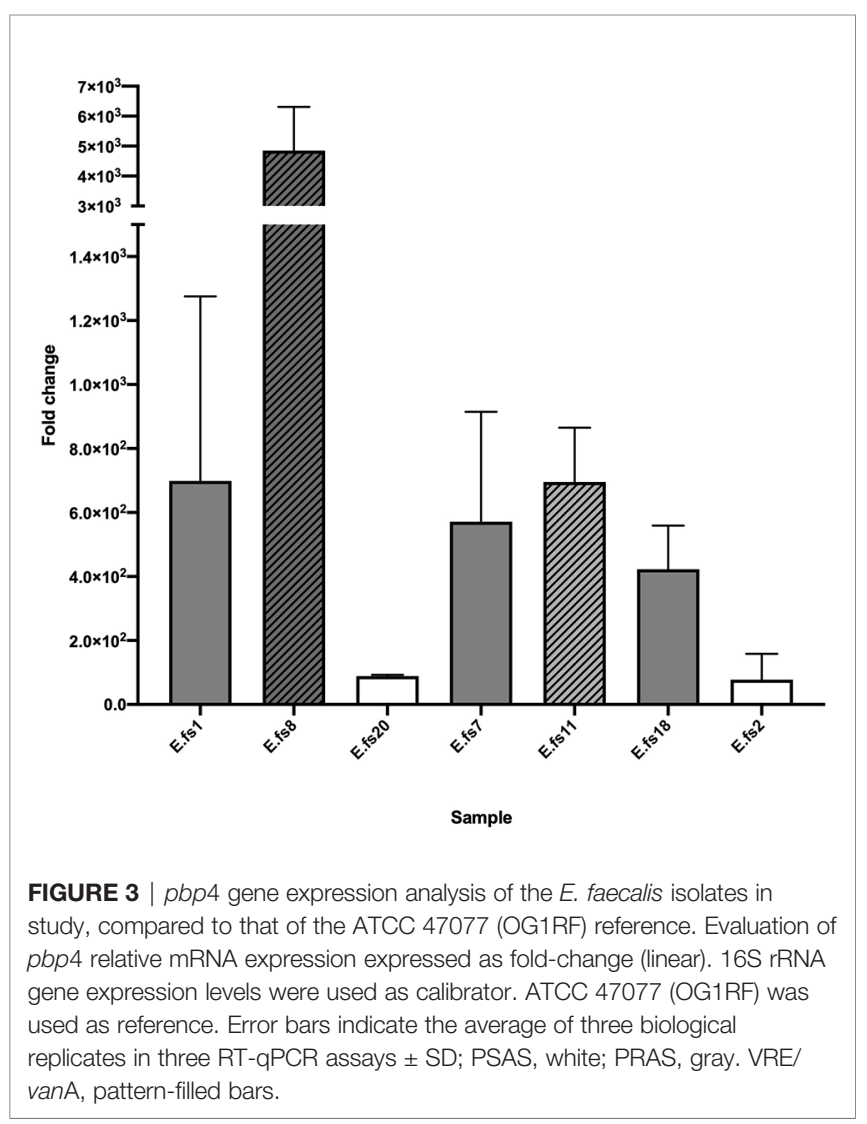

increase compared to that of the ATCC47077 (OG1RF) reference in all BPR-NS strains, demonstrating its role in establishing in vitro non-susceptibility to $\mathrm{BPR}$, although it does not influence bactericidal activity (Figure 3 ).

The only exception was Efs11 (PRAS; CPT-NS; BPR-S), carrying only the D573E substitution. We were not able to demonstrate whether this mutation within the SDN/KTG catalytic sites alone may potentially interfere with the expression of $p b p 4$ transcription, or if the higher upregulation may be related to the $\mathrm{VRE} /$ van $\mathrm{A}$ phenotype, as stated above. Further experiments are needed to confirm its role.

\section{DISCUSSION}

High resistance to penicillins in E. faecalis strains is rare, it can emerge after prolonged $\beta$-lactam therapy treatments (Rice et al., 2018). Nevertheless, the PRAS phenotype was described in several countries (Metzidie et al., 2006; Guardabassi et al., 2010; Conceição et al., 2014), but its epidemiological and clinical impact remains ambiguous, as ampicillin is the treatment of choice for enterococcal infections (Kristich et al., 2014a) and penicillin MIC values were never reported (Mendes et al., 2016). Recently, Kim et al. reported significative differences in mortality rates in patients with a PRAS $E$. faecalis BSI, likely due to the treatment failures of ampicillin and/or piperacillin (Kim et al., 2019).
PBP4, like other PBPs of the same class B (i.e., PBP2a of methicillin-resistant $S$. aureus) performs the cross-linking reaction of the peptidoglycan (PG) biosynthesis, but retains low responsiveness to beta-lactams. Due to its low-reactivity, PBP4 is considered the key basis for intrinsic resistance to cephalosporins in E. faecalis (Arbeloa et al., 2004).

Ceftobiprole, a novel cephalosporin that inhibits the PG cross-linking reaction by acylating the active-site serine of PBPs, maintains a higher affinity for PBP4. Due to its unique ability to target low-affinity PBP4, ceftobiprole is the best candidate as a valid therapeutic option for remarkable $E$. faecalis MDR-phenotypes such as PRAS and VRE. Alterations in this enzyme cause conformational changes that impact the structure of the catalytic motifs (Moon et al., 2018).

This study addressed the mechanism of non-susceptibility to ceftobiprole and the resulting interactions with $E$. faecalis clinical strains, all isolated from bloodstream infections.

A link between benzyl-penicillin resistance and $5^{\text {th }}$ generation cephalosporins non-susceptibility was observed. Our data suggest that this common insensitivity in PRAS isolates results from the involvement of $p b p 4$ mutations in increased gene expression levels and alteration of the penicillin binding domain that could remodel the $\mathrm{PBP} / \beta$ lactam complex.

Our in vitro dynamic data by time-kill curve assays showed that BPR exerts a bactericidal activity against $E$. faecalis isolates despite their MDR phenotypes (VRE, PRAS and BPR-NS), and PBP4 alterations, even after $8 \mathrm{~h}$, consistently with other studies (Werth and Abbott, 2015; Arias et al., 2007). After 24 hours, the bactericidal activity against all isolates - with or without significative $\mathrm{PBP} 4$ changes - was similar, suggesting that ceftobiprole maintains high affinity also for other PBPs.

The majority of the PBP4 mutations found in this study were already reported in the literature, frequently related to PRAS strains (Conceição et al., 2014; Infante et al., 2016; Gawryszewska et al., 2021), but the mechanisms underlying this relationship were not elucidated.

It is well known that the upstream region consensus sequence, in the bacterial promoters, can have an impact on the expression of downstream coding genes (Estrem et al., 1998). In E. faecalis, the involvement of an adenine deletion (delA) upstream of the - 35 region of the $p b p 4$ promoter was recently analyzed in a single strain (Rice et al., 2018). Supported by these observations, we provided a comprehensive analysis on a larger sample of clinical strains belonging to different antibiotic-resistance profiles.

In all BPR-NS, we observed that the delA upstream of the coding sequence results in $p b p 4$ overexpression, hypothetically altering the binding of regulatory proteins. Elevated expression levels may cause increased transpeptidation, resulting in a highly cross-linked peptidoglycan. This demonstrates its role in establishing in vitro non-susceptibility to BPR without affecting its cidal activity.

The combination of delA with additional significant amino acid changes within the PBP4 active sites might result in destabilization and formation of a less competent $\beta$-lactam binding-complex. In one PRAS/BPR-NS strain (Efs1), the 
T418A mutation located 6 amino acids upstream of the catalytic serine included in the ${ }^{424} \mathrm{STFK}^{427}$ motif I affects the MIC value of BPR (BPR $16 \mathrm{mg} / \mathrm{L}$ ), which only remains bactericidal at the highest BPR concentration tested (4X MIC). On the contrary, the I223V mutation located in the N-terminal end, carried by a PSAS/BPR-S strain (Efs20), does not affect the MIC of $\beta$-lactams. This region is known to have no enzymatic function (Infante et al., 2016; Rice et al., 2018; Djoric et al., 2020), and this corroborates the excellent in vitro antibacterial and bactericidal activity of BPR against this strain, exerted at $1 \mathrm{X}, 2 \mathrm{X}$ and $4 \mathrm{X}$ MICs.

We observed that $p b p 4$ was more overexpressed in the VRE/ van A strains regardless of delA in the promoter region (Efs 8 and Efs11) (Figure 3, pattern-filled bars); these strains also reported lower BPR MIC values $(2-4 \mathrm{mg} / \mathrm{L})$. This phenomenon was difficult to explain. In VRE/vanA isolates, the DAla-DLac PG precursor is not processed by $\mathrm{PBP} 4$, as previously reported in $E$. faecium for PBP5 (al-Obeid et al., 1992). We could hypothesize that PBP4 may not work with the modified precursor ending in DLac as it may not be able to identify the target, and this may result in overexpression and subsequent buildup. Besides, production of precursors ending in DAla or in DLac alternatively responsible for resistance to cephalosporins or glycopeptides may promote enhanced cephalosporin susceptibility in the presence of vancomycin/beta-lactam association (Kristich et al., 2014b).

Even though the aim of this study was not to trace E. faecalis epidemiology, we detected three strains carrying PBP4 variants already reported in hospital-associated PRAS strains, epidemiologically related to the High-Risk Enterococcal Clonal Complex (HiRECC) CC87 (Kuch et al., 2012; Gawryszewska et al., 2021). In particular, Efs7 exhibited 4 combined mutations in $\mathrm{PBP} 4$ (designating the $\mathrm{F} 3$ variant), hypothetically responsible for its indifference to $1 \mathrm{X}$ MIC ceftobiprole concentration, in time-kill assays; Efs1, 3 combined mutations (E1 variant); and Efs8, a single mutation (C4 variant). This observation has a clinical and epidemiological impact: the evolution of nosocomial CCs is driven by the acquisition of resistance genes, and the spread of PBP4 variants, responsible for resistance to all betalactams, may potentially compromise the clinical efficacy of $E$. faecalis therapy in hospital settings.

In conclusion, in this study we revealed that benzyl-penicillin and $5^{\text {th }}$ generation cephalosporins interact with PBP4 in similar ways. In PRAS/BPR-NS E. faecalis clinical isolates, the interaction between increased $p b p 4$ gene expression, due to the delA in the upstream region consensus sequence, and the supposed remodeling of the penicillin-binding domain, due to alterations in the PBP4 amino acid sequence, influence their $\beta$-lactams susceptibility profiles without affecting BPR cidal activity.

\section{REFERENCES}

al-Obeid, S., Billot-Klein, D., van Heijenoort, J., Collatz, E., and Gutmann, L. (1992). Replacement of the Essential Penicillin-Binding Protein 5 by HighMolecular Mass PBPs may Explain Vancomycin-Beta-Lactam Synergy in LowLevel Vancomycin-Resistant Enterococcus Faecium D366. FEMS Microbiol. Lett. 70 (1), 79-84. doi: 10.1016/0378-1097(92)90566-7
In the light of the above, we recommend penicillin MICs determination not just for clinical, but also and foremost for epidemiological purposes, to evaluate the spread of isolates belonging this difficult-to-treat epidemic PRAS phenotype as well as to address the proper antimicrobial treatment options for these infections.

The major limitation of this study is the lack of a functional evaluation of delA and amino acid substitutions in PBP4. Further experimental approaches such as whole genome sequence analysis and site-directed mutagenesis, should be attempted to confirm the genetic basis of altered beta-lactams/PBP4 complexes induced by sequence substitutions.

\section{DATA AVAILABILITY STATEMENT}

The data presented in the study are deposited in the GenBank repository (https://www.ncbi.nlm.nih.gov/genbank), under accession numbers from OM032878 to OM032884.

\section{AUTHOR CONTRIBUTIONS}

FC designed the research and directed the project. LL, MC, and FC conducted the research. LL, MC and FC analyzed the data. LL, MC, SS, and FC wrote the manuscript. FC and SS, edited and reviewed the manuscript. All authors read and approved the manuscript.

\section{FUNDING}

This study was partially supported by ADVANZ PHARMA and Programma PIACERI [CovDock]-Linea di intervento 2', University of Catania, Dept. of Biomedical and Biotechnological Sciences (BIOMETEC).

\section{ACKNOWLEDGMENTS}

We would like to thank PharmaTranslated (http://www. pharmatranslated.com/), in particular Dr Silvia Montanari for the language revision. We are grateful to Prof. Daniela Ferrarello and Dr Gino Mongelli for their valuable scientific advice, and the BRIT (Bio-nanotech Research and Innovation Tower) service center of the University of Catania, for the technical support.

\section{SUPPLEMENTARY MATERIAL}

The Supplementary Material for this article can be found online at: https://www.frontiersin.org/articles/10.3389/fcimb.2021. 816657/full\#supplementary-material

Arias, C. A., and Murray, B. E. (2012). The Rise of the Enterococcus: Beyond Vancomycin Resistance. Nat Rev Microbiol 10 (3), 256-78. doi: 10.1038/nrmicro2761.

Arias, C. A., Singh, K. V., Panesso, D., and Murray, B. E. (2007). Evaluation of Ceftobiprole Medocaril Against Enterococcus Faecalis in a Mouse Peritonitis Model. J. Antimicrob. Chemother. 60 (3), 594-8. doi: 10.1093/jac/dkm237

Arbeloa, A., Segal, H., Hugonnet, J. E., Josseaume, N., Dubost, L., Brouard, J. P., et al. (2004). Role of Class A Penicillin-Binding Proteins in PBP5-Mediated 
Beta-Lactam Resistance in Enterococcus Faecalis. J. Bacteriol 186 (5), 12211228. doi: 10.1128/JB.186.5.1221-1228.2004

Bourgogne, A., Garsin, D. A., Qin, X., Singh, K. V., Sillanpaa, J., Yerrapragada, S., et al. (2008). Large Scale Variation in Enterococcus Faecalis Illustrated by the Genome Analysis of Strain OG1RF. Genome Biol. 9, R110. doi: 10.1186/gb2008-9-7-r110

Cabrera, N. L., Malek, A. E., Aitken, S. L., and Arias, C. A. (2020). Outcomes of Patients With Bloodstream Infections Caused by Ampicillin-Susceptible But PenicillinResistant Enterococcus Faecalis: Caution in Interpreting the Results. Antimicrob. Agents Chemother. 64 (4), e02387-e02319. doi: 10.1128/AAC.02387-19

Campanile, F., Bongiorno, D., Mongelli, G., Zanghì, G., and Stefani, S. (2019). Bactericidal Activity of Ceftobiprole Combined With Different Antibiotics Against Selected Gram-Positive Isolates. Diagn Microbiol. Infect. Dis. 93 (1), 77-81. doi: 10.1016/j.diagmicrobio.2018.07.015

Campanile, F., Cassisi, M., Lazzaro, L. M., and Stefani, S. Ceftobiprole Exerts Synergism Against Enterococcus Faecalis Clinical Isolates Despite Their $\beta$ Lactam Resistance. 31st ECCMID, Online 9-12 July 2021. Available at: https://markterfolg.de/ESCMID/Final_Programme_2021/\#page=192.

Clinical and Laboratory Standards Institute (2021). Performance Standards for Antimicrobial Susceptibility Testing, 31th ed. CLSI supplement M100 (ISBN 978-1-68440-104-8 print; ISBN 978-1-68440-105-5 electronic). USA: Clinical and Laboratory Standards Institute.

Conceição, N., da Silva, L. E., Darini, A. L., Pitondo-Silva, A., and de Oliveira, A. G. (2014). Penicillin-Resistant, Ampicillin-Susceptible Enterococcus Faecalis of Hospital Origin: Pbp4 Gene Polymorphism and Genetic Diversity. Infect. Genet. Evol. 28, 289-295. doi: 10.1016/j.meegid.2014.10.018

Conceição, N., Rodrigues, W. F., de Oliveira, K. L. P., da Silva, L. E. P., de Souza, L. R. C., da de Cunha Hueb Barata, Oliveira, C., et al. (2020). Beta-Lactams Susceptibility Testing of Penicillin-Resistant, Ampicillin-Susceptible Enterococcus Faecalis Isolates: A Comparative Assessment of Etest and Disk Diffusion Methods Against Broth Dilution. Ann. Clin. Microbiol. Antimicrob. 19 (1), 43. doi: 10.1186/s12941-020-00386-8

Djorić, D., Little, J. L., and Kristich, C. J. (2020). Multiple Low-Reactivity Class B Penicillin-Binding Proteins Are Required for Cephalosporin Resistance in Enterococci. Antimicrob. Agents Chemother. 64 (4), e02273-e02219. doi: 10.1128/AAC.02273-19

Estrem, S. T., Gaal, T., Ross, W., and Gourse, R. L. (1998). Identification of an UP Element Consensus Sequence for Bacterial Promoters. Proc. Natl. Acad. Sci. U.S.A. 18, 95(17):9761-6. doi: 10.1073/pnas.95.17.9761

Gawryszewska, I., Żabicka, ,. D., Hryniewicz, W., and Sadowy, E. (2021). Penicillin-Resistant, Ampicillin-Susceptible Enterococcus Faecalis in Polish Hospitals. Microb. Drug Resist. 27 (3), 291-300. doi: 10.1089/ mdr.2019.0504

Ghuysen, J. M. (1991). Serine Beta-Lactamases and Penicillin-Binding Proteins. Annu.Rev Microbiol. 45, 37-67. doi: 10.1146/annurev.mi.45.100191.000345

Giacobbe, D. R., Labate, L., Tutino, S., Baldi, F., Russo, C., Robba, C., et al. (2021). Enterococcal Bloodstream Infections in Critically Ill Patients With COVID-19: A Case Series. Ann. Med. 53 (1), 1779-1786. doi: 10.1080/07853890.2021.1988695

Guardabassi, L., Larsen, J., Skov, R., and Schonheyder, H. C. (2010). GentamicinResistant Enterococcus Faecalis Sequence Type 6 With Reduced Penicillin Susceptibility: Diagnostic and Therapeutic Implications. J. Clin. Microbiol. 48, 3820-3821. doi: 10.1128/JCM.01252-10

Hamilton, S. M., Alexander, J. A. N., Choo, E. J., Basuino, L., da Costa, T. M., Severin, A., et al. (2017). High-Level Resistance of Staphylococcus Aureus to $\beta$ Lactam Antibiotics Mediated by Penicillin-Binding Protein 4 (Pbp4). Antimicrob. Agents Chemother. 61 (6), e02727-e02716. doi: 10.1128/ AAC.02727-16

Hollenbeck, B. L., and Rice, L. B. (2012). Intrinsic and Acquired Resistance Mechanisms in Enterococcus. Virulence 5, 421-433. doi: 10.4161/viru.21282

Infante, V. H., Conceição, N., de Oliveira, A. G., and Darini, A. L. (2016). Evaluation of Polymorphisms in Pbp4 Gene and Genetic Diversity in Penicillin-Resistant, Ampicillin-Susceptible Enterococcus Faecalis From Hospitals in Different States in Brazil. FEMS Microbiol. Lett. 363 (7):fnw044. doi: 10.1093/femsle/fnw044

Kim, D., Lee, H., Yoon, E. J., Hong, J. S., Shin, J. H., Uh, Y., et al. (2019). Prospective Observational Study of the Clinical Prognoses of Patients With Bloodstream Infections Caused by Ampicillin-Susceptible But Penicillin-
Resistant Enterococcus Faecalis. Antimicrob. Agents Chemother. 63 (7), e00291-e00219. doi: 10.1128/AAC.00291-19

Kristich, C. J., Djorić, D., and Little, J. L. (2014b). Genetic Basis for VancomycinEnhanced Cephalosporin Susceptibility in Vancomycin-Resistant Enterococci Revealed Using Counterselection With Dominant-Negative Thymidylate Synthase. Antimicrob. Agents Chemother. 58 (3), 1556-1564. doi: 10.1128/ AAC.02001-13

Kristich, C. J., Rice, L. B., and Arias, C. A. (2014a). "Enterococcal Infection Treatment and Antibiotic Resistance," in Enterococci: From Commensals to Leading Causes of Drug Resistant Infection. Eds. M. S. Gilmore, D. B. Clewell, Y. Ike and N. Shankar (Boston: Massachusetts Eye and Ear Infirmary).

Kuch, A., Willems, R. J., Werner, G., Coque, T. M., Hammerum, A. M., Sundsfjord, A., et al. (2012). Insight Into Antimicrobial Susceptibility and Population Structure of Contemporary Human Enterococcus Faecalis Isolates From Europe. J. Antimicrob. Chemother. 67 (3), 551-558. doi: 10.1093/jac/dkr544

Livak, K. J., and Schmittgen, T. D. (2001). Analysis of Relative Gene Expression Data Using Real-Time Quantitative PCR and the $2^{-\Delta \Delta \mathrm{Ct}}$ Method. Methods 25 (4), 402-408. doi: 10.1006/meth.2001.1262

Mendes, R. E., Castanheira, M., Farrell, D. J., Flamm, R. K., Sader, H. S., and Jones, R. N. (2016). Longitudina-14) Analysis of Enterococci and VRE Causing Invasive Infections in European and US Hospitals, Including a Contemporar-13) Analysis of Oritavancin In Vitro Potency. J. Antimicrob. Chemother. 71 (12), 3453-3458. doi: 10.1093/jac/dkw319

Metzidie, E., Manolis, E. N., Pournaras, S., Sofianou, D., and Tsakris, A. (2006). Spread of an Unusual Penicillin- and Imipenem-Resistant But AmpicillinSusceptible Phenotype Among Enterococcus Faecalis Clinical Isolates. J. Antimicrob. Chemother. 57 (1), 158-160. doi: 10.1093/jac/dki427

Moon, T. M., D'Andréa, É.D., Lee, C. W., Soares, A., Jakoncic, J., Desbonnet, C., et al. (2018). The Structures of Penicillin-Binding Protein 4 (PBP4) and PBP5 From Enterococci Provide Structural Insights Into $\beta$-Lactam Resistance. J. Biol. Chem. 293, 18574-18584. doi: 10.1074/jbc.RA118.006052

Ono, S., Muratani, T., and Matsumoto, T. (2005). Mechanisms of Resistance to Imipenem and Ampicillin in Enterococcus Faecalis. Antimicrob. Agents Chemother. 49 (7), 2954-2958. doi: 10.1128/AAC.49.7.2954-2958.2005

Posteraro, B., De Angelis, G., Menchinelli, G., D'Inzeo, T., Fiori, B., De Maio, F., et al. (2021). Risk Factors for Mortality in Adult COVID-19 Patients Who Develop Bloodstream Infections Mostly Caused by Antimicrobial-Resistant Organisms: Analysis at a Large Teaching Hospital in Italy. J. Clin. Med. 10 (8):1752. doi: 10.3390/jcm10081752

Rice, L. B., Desbonnet, C., Tait-Kamradt, A., Garcia-Solache, M., Lonks, J., Moon, T. M., et al. (2018). Structural and Regulatory Changes in PBP4 Trigger Decreased $\beta$-Lactam Susceptibility in. Enterococcus faecalis mBio 3, 9 (2). doi: $10.1128 / \mathrm{mBio} .00361-18$

Rice, L. B., and Murray, B. E. (1995). Beta-Lactamase-Producing Enterococci. In Genetics of Streptococci, Enterococci and Lactococci.: Review of the Six International Conference. Dev. Biol. Stand 85, 107-114.

Sarti, M., Campanile, F., Sabia, C., Santagati, M., Gargiulo, R., and Stefani, S. (2012). Polyclonal Diffusion of Beta-Lactamase-Producing Enterococcus Faecium. J. Clin. Microbio 50 (1), 169-172. doi: 10.1128/JCM.05640-11

Schell, C. M., Tedim, A. P., Rodríguez-Baños, M., Sparo, M. D., Lissarrague, S., Basualdo, J. A., et al. (2020). Detection of $\beta$-Lactamase-Producing Enterococcus Faecalis and Vancomycin-Resistant Enterococcus Faecium Isolates in Human Invasive Infections in the Public Hospital of Tandil, Argentina. Pathogens 9 (2):142. doi: 10.3390/pathogens 9020142

Tan, Y. E., Ng, L. S., and Tan, T. Y. (2014). Evaluation of Enterococcus Faecalis Clinical Isolates With 'Penicillin-Resistant, Ampicillin-Susceptible' Phenotype as Reported by Vitek-2 Compact System. Pathol. 46 (6), 544-550. doi: 10.1097/ PAT. 0000000000000146

The European Committee on Antimicrobial Susceptibility Testing - EUCAST (Valid From 2021-01-01). Version 11.0. Available at: www.eucast.org.

Werth, B. J., and Abbott, A. N. (2015). The Combination of Ampicillin Plus Ceftaroline is Synergistic against Enterococcus faecalis. J. Antimicrob Chemother. 70 (8), 2414-2417. doi: 10.1093/jac/dkv125

White, L. R., Burgess, D. S., Manduru, M., and Bosso, A. J. (1996). Comparison of Three Different In Vitro Methods of Detecting Synergy: Time-Kill, Checkerboard, and E Test. Antimicrob. Agents Chemother. 40 (8), 19141918. doi: 10.1128/AAC.40.8.1914 
Zapun, A., Contreras-Martel, C., and Vernet, T. (2008). Penicillin Binding Proteins and Beta-Lactam Resistance. FEMS Microbiol. Rev. 32 (2), 361-385. doi: 10.1111/j.1574-6976.2007.00095.x

Conflict of Interest: The authors declare that the research was conducted in the absence of any commercial or financial relationships that could be construed as a potential conflict of interest.

Publisher's Note: All claims expressed in this article are solely those of the authors and do not necessarily represent those of their affiliated organizations, or those of the publisher, the editors and the reviewers. Any product that may be evaluated in this article, or claim that may be made by its manufacturer, is not guaranteed or endorsed by the publisher.

Copyright (c) 2022 Lazzaro, Cassisi, Stefani and Campanile. This is an open-access article distributed under the terms of the Creative Commons Attribution License (CC BY). The use, distribution or reproduction in other forums is permitted, provided the original author(s) and the copyright owner(s) are credited and that the original publication in this journal is cited, in accordance with accepted academic practice. No use, distribution or reproduction is permitted which does not comply with these terms. 